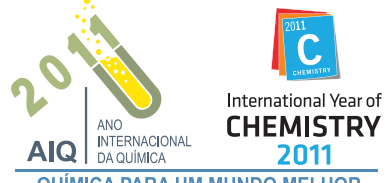

\title{
ÁCIDOS ORGÂNICOS EM AGUARDENTES PRODUZIDAS EM ALAMBIQUE E EM COLUNA
}

Felipe Augusto Thobias Serafim, Silmara França Buchviser, Carlos Alexandre Galinaro e Douglas Wagner Franco*

Instituto de Química de São Carlos, Universidade de São Paulo, CP 780, 13560-970 São Carlos - SP, Brasil

Fernando Valadares Novaes

Escola Superior de Agricultura “Luiz de Queiroz”, Universidade de São Paulo, CP 9, 13418-900 Piracicaba - SP, Brasil

Recebido em 3/12/09; aceito em 5/8/10; publicado na web em 26/11/10

\begin{abstract}
ORGANIC ACIDS IN SUGARCANE SPIRITS' FRACTIONS PRODUCED IN STILLS AND COLUMNS. The concentration of 14 organic acids of 50 sugarcane spirits samples was determined by gas chromatography using flame ionization detection. The organic acids analytical quantitative profile in stills and column distilled spirits from wines obtained from the same must were compared. The comparison was also carried in "head", "heart" and "tail fractions of stills distilled spirits. The experimental data were analyzed by Principal Components Analysis (PCA) and pointed out that the distillation process (stills and column) strongly influences the lead spirits' organic acid composition and that producers' operational "cuts off" to produce "tail", "heart" and "head", fractions should be optimized.
\end{abstract}

Keywords: organic acids; stills; column.

\section{INTRODUÇÃO}

Segundo o Instituto Brasileiro da Cachaça, ${ }^{1}$ as exportações desta bebida cresceram aproximadamente $18 \%$ em valor e $20 \%$ em volume, entre os anos de 2007 e 2008, gerando uma receita de US\$ 16 milhões.

Com o aumento da demanda por bebidas com elevado padrão de qualidade, a adoção de técnicas produtivas mais eficientes é desafio a ser superado pelos produtores. Dentre as etapas de produção da aguardente, a fermentação do mosto e a posterior destilação do vinho são as mais importantes para a obtenção de uma bebida com qualidades químicas e sensoriais superiores. ${ }^{2.3}$

A produção de aguardente em larga escala é realizada em colunas de aço inoxidável, onde não há separação do destilado em frações. Trata-se de um processo contínuo de destilação em que a entrada do mosto fermentado (vinho) na coluna e as respectivas saídas do destilado e do vinhoto ocorrem simultaneamente. Neste caso, de cada tonelada de cana-de-açúcar processada podem ser obtidos de 140 a $170 \mathrm{~L}$ de aguardente a $47,5 \%$ de etanol em volume, a $20^{\circ} \mathrm{C}$, segundo o grau de maturação da cana-de-açúcar e as consequentes eficiências nas fases de extração do caldo, fermentação do mosto e destilação do vinho.

Na produção da aguardente em pequena escala, a destilação ocorre em batelada nos denominados alambiques. Durante o processo, o destilado é separado em três diferentes frações. A primeira é denominada "cabeça" e seu teor alcoólico varia entre 55 e $65 \%$ em volume, a $20^{\circ} \mathrm{C}$, sendo o volume coletado correspondente a 5 a $10 \%$ do volume total destilado. A segunda fração, o "coração" (parte nobre do destilado), possui teor alcoólico entre 43 a $45 \%$ em volume, a $20^{\circ} \mathrm{C}$, correspondendo a 75 a $80 \%$ do volume destilado; e, a última fração, denominada "cauda" ou "água fraca", começa a ser coletada quando o teor alcoólico do destilado que flui na bica do alambique é da ordem de $38 \%$ em volume, a $20{ }^{\circ} \mathrm{C}$, estendendo-se até que o seu volume atinja cerca de $10 \%$ do destilado total produzido. Nestas pequenas unidades, a cada tonelada de cana processada podem ser obtidos de 80 a 120 L de aguardente (fração "coração"), em função também da eficiência daquelas três fases operacionais do processo. ${ }^{4-6}$

Embora a fermentação e a destilação tenham sido introduzidas

*e-mail: douglas@iqsc.usp.br empiricamente no país há quase 5 séculos, ${ }^{5}$ não existe na prática, principalmente junto aos pequenos produtores, uma uniformidade quanto à condução do processo fermentativo e, tampouco, em relação ao critério utilizado na separação das frações dos seus destilados em alambique.

Dentre os compostos secundários que são formados durante a etapa de fermentação do mosto, destacam-se os ácidos orgânicos. Estes exercem forte influência sobre a qualidade e as propriedades sensoriais das bebidas e na estabilidade do mosto durante a etapa fermentativa. ${ }^{7-12}$ As moléculas dos ácidos podem penetrar nas células dos micro-organismos por difusão simples, interferindo no metabolismo das mesmas, alterando a reposição e a absorção de nutrientes e o crescimento celular, promovendo assim, certo controle biológico. ${ }^{12} \mathrm{~A}$ presença de seus respectivos ésteres confere à bebida características sensoriais diferenciadas, agregando a ela aromas e sabores frutados e achocolatados. ${ }^{13-15}$

A presença dos ácidos orgânicos no destilado está também relacionada aos cuidados dispensados durante a produção da bebida. Os ácidos acético e lático geralmente estão associados à contaminação do mosto por micro-organismos. ${ }^{16-18}$ Portanto, ambos podem ser indicadores dos controles antisséptico e de temperatura utilizados pelos produtores no decorrer do processo fermentativo. Já a presença de ácidos graxos no destilado deve-se à sua formação na etapa aeróbica do processo, durante a qual a levedura sintetiza a biomassa para seu crescimento populacional. ${ }^{19} \mathrm{~A}$ falta de uma filtração eficiente do vinho pode permitir um aumento do teor de leveduras presentes no interior do destilador, o que resultará no aumento da concentração de ácidos graxos no destilado. ${ }^{20}$

Neste trabalho, tomando-se como indicadores do processo os ácidos orgânicos (lático, pirúvico, oxálico, malônico, succínico, glutárico, cáprico, citramálico, málico, láurico, cítrico, mirístico e palmítico), buscou-se conhecer o perfil dos mesmos nas três diferentes frações resultantes do processo de produção de aguardentes em alambique, assim como no destilado único produzido em coluna, para então se obter mais informações sobre o fracionamento do destilado. Para tanto, os resultados analíticos referentes às concentrações dos ácidos orgânicos foram analisados por métodos quimiométricos utilizando, neste caso, a análise de componentes principais (PCA - Principal Component Analysis), através do software Minitab 15 English Statistical. 


\section{PARTE EXPERIMENTAL}

Foram determinados os perfis dos ácidos orgânicos nas três frações do destilado (cabeça, coração e cauda) em 15 amostras de aguardente de cana produzidas em alambique, provenientes de diferentes produtores. Cinco destas amostras, oriundas do mesmo mosto fermentado de caldo de cana (vinho), foram submetidas à destilação em alambique e em coluna. Os reagentes e os solventes utilizados foram sempre de grau analítico (Fluka, Sigma Aldrich) e de grau HPLC (Merck, Mallinckrdt), respectivamente. Nas diluições e no preparo das soluções, foi utilizada água deionizada, obtida em sistema Milli-Q (Millipore).

O método de análise dos ácidos orgânicos foi adaptado da literatura. ${ }^{21-24}$ Após pré-concentração através da secagem completa de $20 \mathrm{~mL}$ de amostra à temperatura ambiente, efetuou-se a adição de $200 \mu \mathrm{L}$ de solução derivatizante $(100 \mu \mathrm{L}$ de 2,2,2-trifluoro-Nmethyl-N-trimethylsilyl-acetamide(MSTFA)/100 $\mu \mathrm{L}$ de solução de ácido nonanoico em $\mathrm{ACN}$ ). $\mathrm{O}$ volume de $1 \mu \mathrm{L}$ desta solução foi injetado no cromatógrafo de fase gasosa (CG) Hewlett-Packard modelo 5890, equipado com detector de ionização por chama (Flame Ionization Detection - FID), com coluna capilar DB-5 (5\%-Phenylmethylpolysiloxane) com dimensões de $50 \mathrm{~m}$ x 0,20 $\mathrm{mm}$ x $0,33 \mu \mathrm{m}$. A quantificação foi realizada com o auxílio de curva de calibração e padrão interno (ácido nonanoico). A confirmação de cada um dos analitos foi efetuada por adição de padrões e por comparação dos tempos de retenção relativos das amostras com os dos padrões analíticos, nas mesmas condições experimentais. Para a análise do ácido acético, utilizou-se o método analítico descrito por Boscolo et al.. ${ }^{25}$

As concentrações dos ácidos orgânicos presentes nas amostras foram finalmente submetidas à análise de componentes principais, utilizando-se o software Minitab 15 English Statistical.

\section{RESULTADOS E DISCUSSÃO}

O perfil cromatográfico de uma solução contendo os padrões dos derivados de ácidos orgânicos em uma amostra de aguardente e os correspondentes perfis cromatográficos típicos obtidos para as três frações do destilado podem ser observados na Figura 1S, material suplementar.

Em trabalhos anteriores ${ }^{9,26}$ foram determinadas as concentrações de ácidos orgânicos em aguardentes obtidas no comércio. Neles foram reportados os ácidos acético, propiônico, isobutírico, n-butírico, isovalérico, n-valérico, iso-caproico, n-caproico, n-heptanoico, n-caprílico, cáprico, láurico, mirístico e palmítico. Estes quatro últimos também foram determinados neste trabalho e apresentaram teores médios inferiores aos encontrados na literatura (Tabela 1), provavelmente devido ao fato das amostras aqui estudadas serem recém-destiladas e não envelhecidas, já que, como se sabe, o envelhecimento em recipientes de madeira resulta no aumento da acidez total das aguardentes, devido à incorporação dos ácidos orgânicos extraídos da madeira do tonel. ${ }^{27-30}$

Tabela 1. Teores médios $\left(\mathrm{mg} / 100 \mathrm{~mL}\right.$ de $\left.\mathrm{AA}^{\mathrm{a}}\right)$ dos ácidos graxos $(\mathrm{C} 2$, C10-C16) nas amostras de aguardentes da literatura ${ }^{10}$ e dos destilados de alambique e de coluna

\begin{tabular}{lccccc}
\hline Ácidos & $\begin{array}{c}\text { Aguardente } \\
\text { tipo } \mathrm{A}^{\mathrm{b}}\end{array}$ & $\begin{array}{c}\text { Aguardente } \\
\text { tipo B }^{\mathrm{b}}\end{array}$ & $\begin{array}{c}\text { Aguardente } \\
\text { tipo } \mathrm{C}^{\mathrm{b}}\end{array}$ & Alambique & Coluna \\
\hline acético (C2) & 57,5 & 99,2 & 72,8 & 79,3 & 62,9 \\
cáprico(C10) & 2,0 & 1,60 & 1,37 & 0,20 & 0,78 \\
láurico (12) & 0,73 & 0,60 & 0,54 & 0,12 & 0,13 \\
mirístico (C14) & 0,52 & 0,40 & 0,37 & 0,07 & 0,03 \\
palmítico (C16) & 1,10 & 0,39 & 0,55 & 0,31 & 0,22 \\
\hline
\end{tabular}

As presenças dos ácidos malônico, glutárico, málico e cítrico não foram detectadas pela metodologia utilizada, indicando que suas concentrações são inferiores aos respectivos limites de detecção (Tabela 2). Apesar de constatada a presença do ácido oxálico nas amostras, sua quantificação não foi possível devido à instabilidade térmica do derivado silânico (Oxálico-TMS ${ }_{2}$ ), problema já anteriormete reportado na literatura. ${ }^{21} \mathrm{~A}$ ausência do ácido málico também pode ser devida à fermentação malolática, que converte o ácido málico em ácido lático, diminuindo assim, o teor desse ácido. ${ }^{31}$

\section{Aguardente de alambique e de coluna}

A comparação entre os perfis dos ácidos orgânicos das aguardentes produzidas em alambique e em coluna foi baseada nos resultados de 5 amostras de aguardente de procedência diversa e cujos vinhos foram divididos em duas partes, sendo uma destilada em alambique e a outra em coluna. Como pode ser observado na Figura 1, as concentrações dos ácidos mirístico, glicólico, palmítico, citramálico, lático e acético foram superiores nas amostras destiladas em alambique com relação àquelas provenientes de coluna. $\mathrm{O}$ contrário se observa com respeito aos ácidos succínico, cáprico e láurico. Tal fato pode ser explicado principalmente pela grande diferença existente na geometria dos aparelhos de destilação empregados no processo de destilação, já que, enquanto o alambique possui apenas um estágio de destilação, a coluna é constituída por 15 a 20 estágios $^{32}$ sobrepostos e interligados entre si.

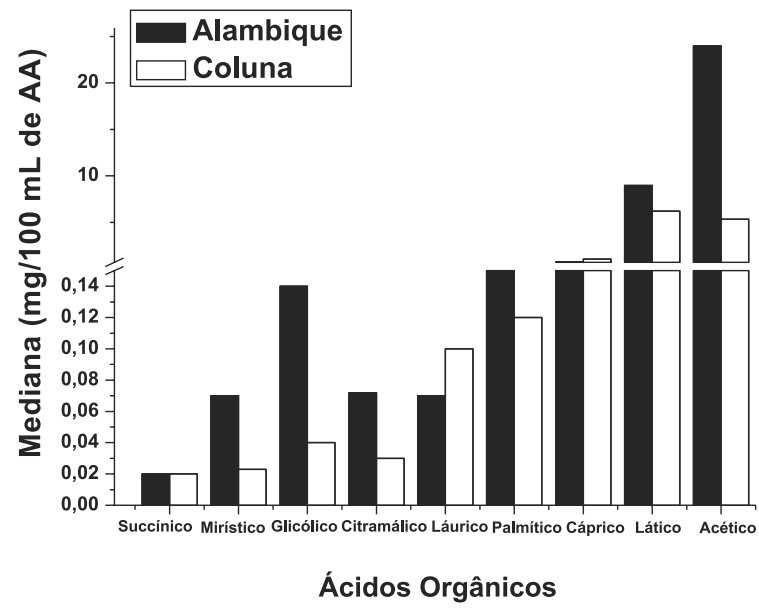

Figura 1. Comparação dos valores de medianas de concentração dos ácidos orgânicos presentes nos destilados de alambique (coração) e de coluna em amostras oriundas do mesmo mosto fermentado

No caso da produção do destilado em alambiques, além do seu fracionamento em três diferentes porções (cabeça, coração e cauda), o equilíbrio do efeito conjugado de alguns parâmetros tais como teor alcoólico do vinho, temperatura de destilação (entre 85 e $96{ }^{\circ} \mathrm{C}$ ), geometria e material de construção do aparelho, diâmetro da saída dos vapores (início da alonga), sistema de resfriamento no topo da coluna (capelo ou deflegmador) ${ }^{33}$ e teor alcoólico do destilado final também devem ser levados em consideração. Esta conjugação de fatores certamente influi na composição química do destilado.

Com o objetivo de verificar similaridades na composição química da bebida, conforme já observado em outros trabalhos, ${ }^{34-36}$ aplicou-se a análise multivariada aos teores de ácidos orgânicos presentes nos destilados de alambique e de coluna. A análise de componentes principais aplicada aos resultados que compõem a Figura 1 deu origem aos gráficos de scores e de loading, apresentados nas Figuras 2 e 3, respectivamente. 
Tabela 2. Valores dos limites de detecção da metodologia, das médias e medianas de concentração (mg/100 mL de A.A) dos ácidos analisados nas frações de "cabeça", "coração" e "cauda" do destilado

\begin{tabular}{|c|c|c|c|c|c|c|c|c|}
\hline \multirow[b]{2}{*}{$\begin{array}{l}\text { Ácidos } \\
\text { orgânicos }\end{array}$} & \multirow[b]{2}{*}{$\begin{array}{l}\text { Limite de detecção } \\
(\mathrm{ppb})\end{array}$} & \multirow[b]{2}{*}{$\begin{array}{c}\text { Recuperação } \\
(\%)\end{array}$} & \multicolumn{2}{|c|}{ Cabeça } & \multicolumn{2}{|c|}{ Coração } & \multicolumn{2}{|c|}{ Cauda } \\
\hline & & & Média & Mediana & Média & Mediana & Média & Mediana \\
\hline Lático & 25,0 & 60 & 5,11 & 2,98 & 11,0 & 8,97 & 39,2 & 23,8 \\
\hline Glicólico & 50,0 & 72,3 & $1,40 \times 10^{-1}$ & $1,30 \times 10^{-1}$ & $1,90 \times 10^{-1}$ & $1,40 \times 10^{-1}$ & 1,56 & $2,20 \times 10^{-1}$ \\
\hline Pirúvico & 50,0 & 12 & $4,00 \times 10^{-2}$ & $4,00 \times 10^{-2}$ & $2,10 \times 10^{-1}$ & $2,00 \times 10^{-1}$ & $5,00 \times 10^{-1}$ & $3,70 \times 10^{-1}$ \\
\hline Succínico & 25,0 & 97 & $2,40 \times 10^{-2}$ & $1,80 \times 10^{-2}$ & $4,00 \times 10^{-2}$ & $2,00 \times 10^{-2}$ & $1,80 \times 10^{-1}$ & $5,00 \times 10^{-2}$ \\
\hline Citramálico & 100 & 80 & $4,40 \times 10^{-2}$ & $4,20 \times 10^{-2}$ & $7,60 \times 10^{-2}$ & $7,20 \times 10^{-2}$ & $1,30 \times 10^{-1}$ & $1,10 \times 10^{-1}$ \\
\hline Cáprico & 250 & 18 & 2,54 & 1,02 & $7,30 \times 10^{-1}$ & $4,60 \times 10^{-1}$ & $6,00 \times 10^{-1}$ & $2,10 \times 10^{-1}$ \\
\hline Láurico & 50,0 & 93 & $4,70 \times 10^{-1}$ & $2,78 \times 10^{-1}$ & $1,20 \times 10^{-1}$ & $7,00 \times 10^{-2}$ & $1,40 \times 10^{-1}$ & $9,00 \times 10^{-1}$ \\
\hline Mirístico & 5,00 & 93 & $1,70 \times 10^{-1}$ & $1,60 \times 10^{-1}$ & $7,00 \times 10^{-2}$ & $7,00 \times 10^{-2}$ & $1,00 \times 10^{-1}$ & $7,90 \times 10^{-2}$ \\
\hline Palmítico & 50,0 & 95 & $7,00 \times 10^{-1}$ & $4,80 \times 10^{-1}$ & $3,10 \times 10^{-1}$ & $3,00 \times 10^{-1}$ & $2,20 \times 10^{-1}$ & $2,00 \times 10^{-1}$ \\
\hline Acético & 5,00 & 99.9 & 126 & 9,36 & 95,3 & 24,0 & 312 & 45,0 \\
\hline Málico & 25,0 & 87 & $<\mathrm{LD}^{*}$ & $<\mathrm{LD}$ & $<\mathrm{LD}$ & $<\mathrm{LD}$ & $<\mathrm{LD}$ & $<\mathrm{LD}$ \\
\hline Glutárico & 25,0 & 102 & $<\mathrm{LD}$ & $<\mathrm{LD}$ & $<\mathrm{LD}$ & $<\mathrm{LD}$ & $<\mathrm{LD}$ & $<\mathrm{LD}$ \\
\hline Cítrico & 200 & 88 & $<\mathrm{LD}$ & $<\mathrm{LD}$ & $<\mathrm{LD}$ & $<\mathrm{LD}$ & $<\mathrm{LD}$ & $<\mathrm{LD}$ \\
\hline Malônico & $1,00 \times 10^{3}$ & 98 & $<\mathrm{LD}$ & $<\mathrm{LD}$ & $<\mathrm{LD}$ & $<\mathrm{LD}$ & $<\mathrm{LD}$ & $<\mathrm{LD}$ \\
\hline
\end{tabular}

$*<\mathrm{LD}=$ Menor que o Limite de Detecção

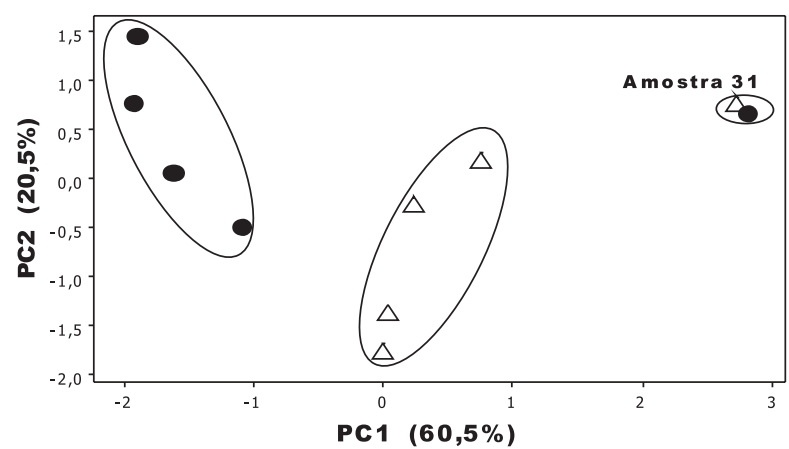

Figura 2. Gráfico de scores da análise de componentes principais ( $\bullet$ coluna; $\triangle$ alambique)

A diferença quantitativa dos teores de ácidos orgânicos, observada nos destilados da fração coração do alambique e do produto de coluna, explica a formação dos dois grupos observados no gráfico de scores.

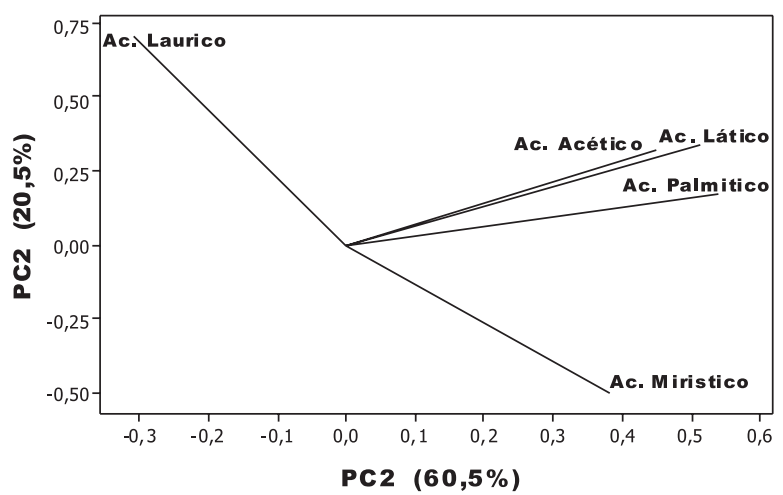

Figura 3. Gráfico de loading da análise de componentes principais

O gráfico de loading indica que dentre os 9 ácidos analisados, os ácidos acético, lático, mirístico, láurico e palmítico apresentam características que permitem a distinção entre os dois tipos de destilados, explicando através das somas das duas primeiras componentes principais $81,0 \%$ da variância do conjunto dos dados originais (PC1 $=60,5 \%$ × PC2 $=20,5 \%$ ).

Nota-se ainda na Figura 2 a presença de um outlier correspondente à amostra 31, comportamento que pode ser explicado considerandose as elevadas concentrações de ácidos acético e lático, valores que indicam que a etapa fermentativa e sua posterior destilação podem não ter sido conduzidas de maneira apropriada. ${ }^{37}$

\section{Perfil dos ácidos orgânicos nas frações cabeça, coração e cauda}

Foram elaborados os perfis quali e quantitativo dos ácidos orgânicos presentes nas três frações - cauda, coração e cabeça - obtidas no processo de destilação em alambique, de 15 amostras de aguardentes coletadas diretamente dos alambiques dos diferentes produtores. $\mathrm{Na}$ Figura 4 estão apresentados os valores das medianas de concentração destes ácidos nas referidas frações.

Como se pode observar, as concentrações dos ácidos acético, succínico, glicólico, citramálico e lático são muito superiores na fração cauda com relação às outras duas. Tal comportamento pode estar associado ao caráter hidrofílico ${ }^{38}$ mais acentuado desses compostos, uma vez que o teor de água na fração cauda (aproximadamente $85 \%$ ) é bem superior às demais.

No caso dos ácidos cáprico, láurico, mirístico e palmítico, cujas solubilidades em etanol são maiores do que na água, ${ }^{38}$ as concentrações encontradas foram superiores na fração cabeça, com maior teor alcoólico (65\%).

A composição em alcoóis superiores e de aldeídos no destilado apresentou uma distribuição ao longo das três frações do destilado de alambique coerente com suas respectivas solubilidades em água e etanol ${ }^{39} \mathrm{e}$, mais propriamente, de acordo com seus respectivos coeficientes de purificação, ${ }^{40}$ comportamento semelhante ao observado na distribuição dos ácidos orgânicos.

A aplicação da análise de componentes principais aos resultados obtidos originou os gráficos de scores e de loading apresentados, res- 


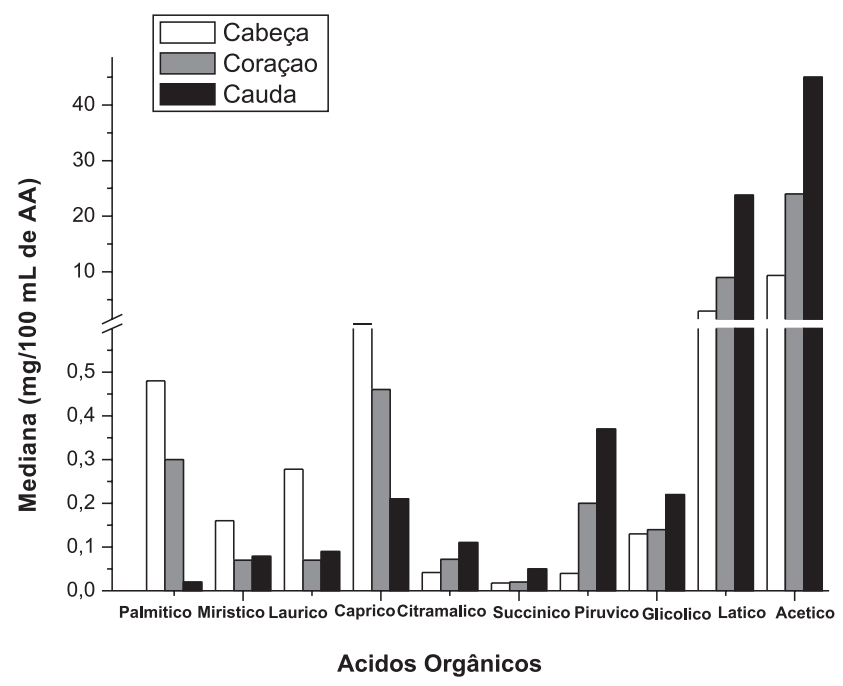

Figura 4. Valores de medianas de concentração para os teores de ácidos orgânicos presentes nas três frações dos destilados de alambique, para 15 amostras distintas

pectivamente, nas Figuras 5 e 6 . A soma dos primeiras componentes principais (PC1 + PC2) permite explicar 91,3\% da variação dos dados originais, permitindo observar-se (Figura 5) uma tendência à formação de três grupos representados pelas frações de cauda, coração e cabeça.

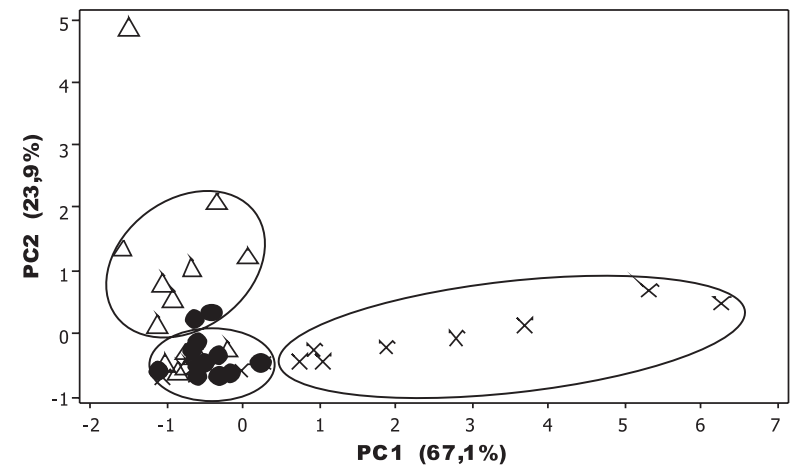

Figura 5. Gráfico de scores da análise de componentes principais (X cabeça; - coração; $\triangle$ cauda)

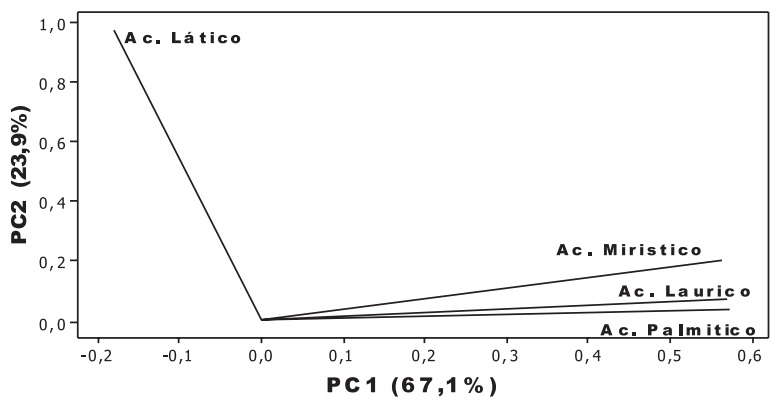

Figura 6. Gráfico de loading da análise de componentes principais (PC1 x P2)

Com o gráfico de loading, Figura 6, verifica-se também, através do componente PC1 $(67,4 \%)$, que o ácido lático e os ácidos láurico, mirístico e palmítico são os principais responsáveis pela distinção observada entre as frações cauda e cabeça, respectivamente. No caso da PC2 $(23,9 \%)$ também se observa acentuada influência do ácido lático na diferenciação de parte das amostras relativas às frações de cauda e coração.
O grupo correspondente à fração coração foi o que apresentou menor dispersão, contendo amostras correspondentes às outras duas frações (outliers). Isso pode ser consequência dos outliers presentes na fração coração, mas pertencentes à fração cauda, apresentarem um valor da mediana da concentração de $11,1 \mathrm{mg} / 100 \mathrm{~mL}$ de A.A para o ácido lático, valor este muito próximo ao valor da mediana das amostras da fração coração $(8,75 \mathrm{mg} / 100 \mathrm{~mL}$ de A.A). Para os outliers oriundos da fração cabeça, o valor da mediana da concentração dos ácidos palmítico e mirístico foi de 0,22 e 0,06 mg/100 mL de A.A, respectivamente, enquanto que para as amostras da fração coração foi de 0,30 e $0,07 \mathrm{mg} / 100 \mathrm{~mL}$ de AA.

As amostras responsáveis pela formação do grupo correspondente à fração cauda (Figura 5) apresentaram um valor da mediana da concentração de 54,6 mg/100 mL de AA para o ácido lático. Já as amostras responsáveis pela formação do grupo da fração cabeça apresentaram um valor de mediana de 0,70 e 0,14 mg/100 mL de AA para os ácidos palmítico e mirístico, respectivamente. Como pôde ser observado, os valores de mediana de concentração para os respectivos descritores das frações de cauda e cabeça são muito superiores aos observados para as amostras que compõem o grupo da fração coração.

Assim, no que diz respeito ao perfil dos ácidos orgânicos nas frações, nas aguardentes produzidas em alambique, observa-se que parte do destilado que é descartado (frações cauda e cabeça) possui perfil semelhante ao "coração" do destilado.

\section{CONCLUSÕES}

No tocante aos teores de ácidos orgânicos, a aplicação da análise das componentes principais aos resultados analíticos para os destilados de alambique e de coluna (oriundos do mesmo vinho) evidencia que o tipo do aparelho de destilação influencia na composição quantitativa dos ácidos orgânicos da bebida. Tal ferramenta quimiométrica também permitiu distinguir os destilados cujos teores de ácidos lático e acético se mostraram muito acima da média, indicando uma provável fermentação mal conduzida. Nota-se também que, embora exista uma tendência de distinção entre as frações de "cabeça", "cauda" e "coração" do destilado de alambique, esta distinção ainda deixa muito a desejar e que as condições experimentais para se realizar os "cortes" durante o processo de destilação em alambique necessitam de uma melhor padronização.

Para uma análise mais criteriosa sobre a melhor maneira de se realizar os "cortes" durante a destilação em alambiques, há a necessidade de se observar também a composição química das frações com respeito aos demais compostos que compõem o destilado.

\section{MATERIAL SUPLEMENTAR}

No material suplementar, disponível em http://quimicanova. sbq.org.br, na forma de arquivo PDF, com acesso livre, encontramse o perfil cromatográfico dos derivados silânicos dos ácidos orgânicos para os padrões analíticos e cada fração do destilado de alambique (Figura 1S), a concentração dos ácidos orgânicos nas amostras de aguardentes da fração coração de alambiques e de colunas oriundas do mesmo vinho. (Tabela 1S), bem como o resultado das três frações do destilado de alambique (Tabela $2 \mathrm{~S}$ ), além dos valores médios e de mediana dos quatro descritores das amostras correspondentes a cada fração do destilado e dos seus outliers (Tabela 3S).

\section{AGRADECIMENTOS}

Ao CNPq, à CAPES e à FAPESP pelo apoio financeiro. 


\section{REFERÊNCIAS}

1. http://www.ibrac.net, acessada em Outubro 2009 e Novembro 2010.

2. Reche, R. V.; Leite-Neto, A. F.; Silva, A. A.; Galinaro, C. A.; De Osti, R. Z.; Franco, D. W.; J. Agric. Food Chem. 2007, 55, 6603.

3. Nascimento, R. F.; Cardoso, D. R.; Lima-Neto, B. S.; Faria, J. B.; Franco, D. W.; Quim. Nova 1998, 21, 735.

4. Mutton, M. J. R.; Mutton, M. A. Em Tecnologia de bebidas: matéria prima, processamento, BPF/APPCC, legislação e mercado; Venturini, W. G. F., ed.; $1^{\text {a }}$ ed., Edgard Blucher: São Paulo, 2005.

5. Lima, U. A.; Aguardente: fabricação em pequenas destilarias, $1^{\mathrm{a}}$ ed., FEALQ: Piracicaba, 1999.

6. Bizelli, L. C.; Ribeiro, C. A. F.; Novaes F. V.; Scientia Agricola 2000, 57,623 .

7. Giasi, P.; Horne, J.; Lawless, H. T.; Chemical Senses 1996, 21, 397.

8. Lay-Kenow, N.; Anal. Chim. Acta 2002, 465, 309.

9. Nascimento, R. F; Cardoso, D. R.; Lima-Neto, B. S. N.; Franco, D. W.; Chromatographia 1998, 48, 751.

10. Campos, F. M.; Figueiredo, A. R.; Hogg, T. A.; Couto, J. A.; Food Microbiol. 2009, 26, 409.

11. Yang, Z.; Fang, Q.; Nout, R. M. J.; Ji, B.; Zhou, F.; Zhang, H.; Eur. Food Res. Technol. 2008, 227, 1183.

12. Azeredo, H. M. C.; Fundamentos de Estabilidade de Alimentos, $1^{\mathrm{a}}$ ed., Técnica: Fortaleza, 2004, cap. 5.

13. Burdock, G. A.; Fenaroli's Handbook of Flavor Ingredients, $4^{\text {th }}$ ed.; CRC Press: Washington, 2001.

14. Goss, K. A.; Alharethi, R.; Laposata, M.; Alcohol 1999, 17, 241.

15. Nascimento, E. S. P.; Cardoso, D. R.; Franco, D. W.; Agric. Food Chem. 2008, 56, 5488 .

16. Schwan, R. F.; Mendonça, A. T ; Da Silva, J. J. ; Rodrigues, V.; Wheals, A.; Antonie van Leeuwenhoek 2001, 79, 89.

17. Costa, V. M.; Basso, T. O.; Angeloni, L. H. P.; Oetterer, M.; Basso L. C.; Ciênc. Agrotec 2008, 32, 503

18. Carvalho-Netto, O. V.; Rosa, D. D.; Camargo, L. E. A.; Scientia Agricola 2008, 65, 508

19. Nykänen, L.; Nykänen, I. Em Distilled Beverages; Maarse, H., ed.; Marcel Dekker: New York, 1991, cap. 15.

20. Suomalainen, H.; Nykänen, L.; Eriksson, K.; Am. J. Enology and Viticulture 1974, 25, 179 .
21. Lay-Keow, Ng.; Lafontaine, P.; Harnois J.; J. Chromatogr. 2000, 873, 29.

22. Park, Y. J.; Kim, K. R.; Kim, J. H.; J. Agric. Food Chem. 1999, 47, 2322.

23. Lay-Keow, Ng.; Hupe, M.; Vanier, M.; Moccia, D.; J. Agric. Food Chem. 2001, 49, 1132 .

24. Blau, K.; Halket, J. Em Handbook of Derivatives for Chromatography; Evershed, R. P., ed.; Wiley: Chichester, 1993, cap. 5.

25. Boscolo, M.; Bezerra, C. W B.; Cardoso, D. R.; Lima Neto, B. S.; Franco, D. W.; J. Braz. Chem. Soc. 2000, 11, 86.

26. Nascimento, R. F ; Cardoso, D. R. ; Keukeleire, D. ; Lima N., B. S ; Franco, D. W.; J. Agric. Food Chem. 2000, 48, 6070.

27. Nykänen, L.; Am. J. Enology and Viticulture 1986, 37, 84.

28. Lee, K. -Y. M.; Paterson, A.; Piggott, J. R.; Richardson, G. D.; J. Institute of Brewing 2001, 107, 287.

29. Conner, J. M.; Paterson, D. A.; Birkmyre, L; Piggott, J. R.; J. Institute of Brewing 1998, 105, 287.

30. Baldwin, S.; Black, A. R.; Andreasen, A. A.; Adams, S. L.; J. Agric. Food Chem. 1967, 15, 381

31. Lea, A. G. H.; Piggott, J. R.; Fermented Beverage Production, $2^{\text {nd }}$ ed., Kluwer Academic/Plenum Publishers: New York, 2003.

32. Reche, R. V.; Neto, A. F. L.; Silva, A. A.; Galinaro, C. A.; De Osti, R. Z.; Franco D. W.; J. Agric. Food Chem. 2007, 55, 6603.

33. Maia, A. B. R. A.; Curso de Destilação da Cachaça, Vassouras, 2000, cap. 5 .

34. Galinaro, C. A.; Franco, D. W.; Quim. Nova 2009, 32, 1447.

35. Sampaio, O. M.; Reche, R. V.; Franco, D. W.; J. Agric. Food Chem. 2008, 56, 1661 .

36. Silva, A. A.; Nascimento, E. S. P.; Cardoso, D. R.; Franco, D. W.; J. Sep. Sci. 2009, 32, 1.

37. Cardozo, R. M. D.; Martins, J. F. P.; França, A.; Resumos da III Jornada Nacional da Agroindústria, Bananeiras, Brasil, 2008.

38. Weast, R. C.; Handbook of Chemistry and Physics, $58^{\text {th }}$ ed., CRC Press: Florida, 1978.

39. Galinaro, C. A.; Silva, A. A.; Cardoso, D. R.; Franco D. W.; Resumos da $31^{a}$ Reunião Anual da Sociedade Brasileira de Química, Águas de Lindóia, Brasil, 2008.

40. Novaes, F. V.; Noções Básicas Sobre a Teoria da Destilação Alcoólica, ESALQ/USP, Piracicaba, 1994, publicação interna. 


\section{ÁCIDOS ORGÂNICOS EM AGUARDENTES PRODUZIDAS EM ALAMBIQUE E EM COLUNA}

Felipe Augusto Thobias Serafim, Silmara França Buchviser, Carlos Alexandre Galinaro e Douglas Wagner Franco* Instituto de Química de São Carlos, Universidade de São Paulo, CP 780, 13560-970 São Carlos - SP, Brasil Fernando Valadares Novaes

Escola Superior de Agricultura "Luiz de Queiroz", Universidade de São Paulo, CP 9, 13418-900 Piracicaba - SP, Brasil

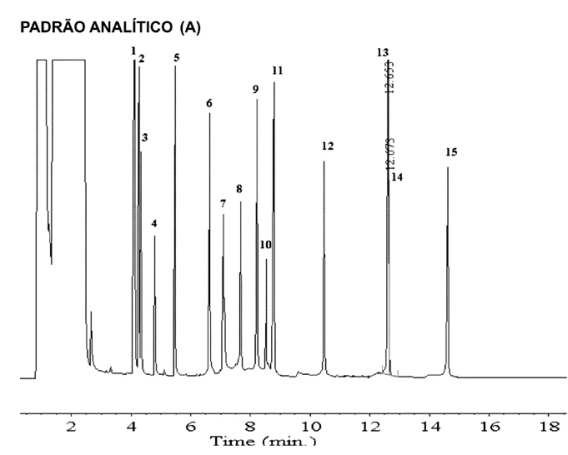

FRAÇÃo CABEÇA (B)
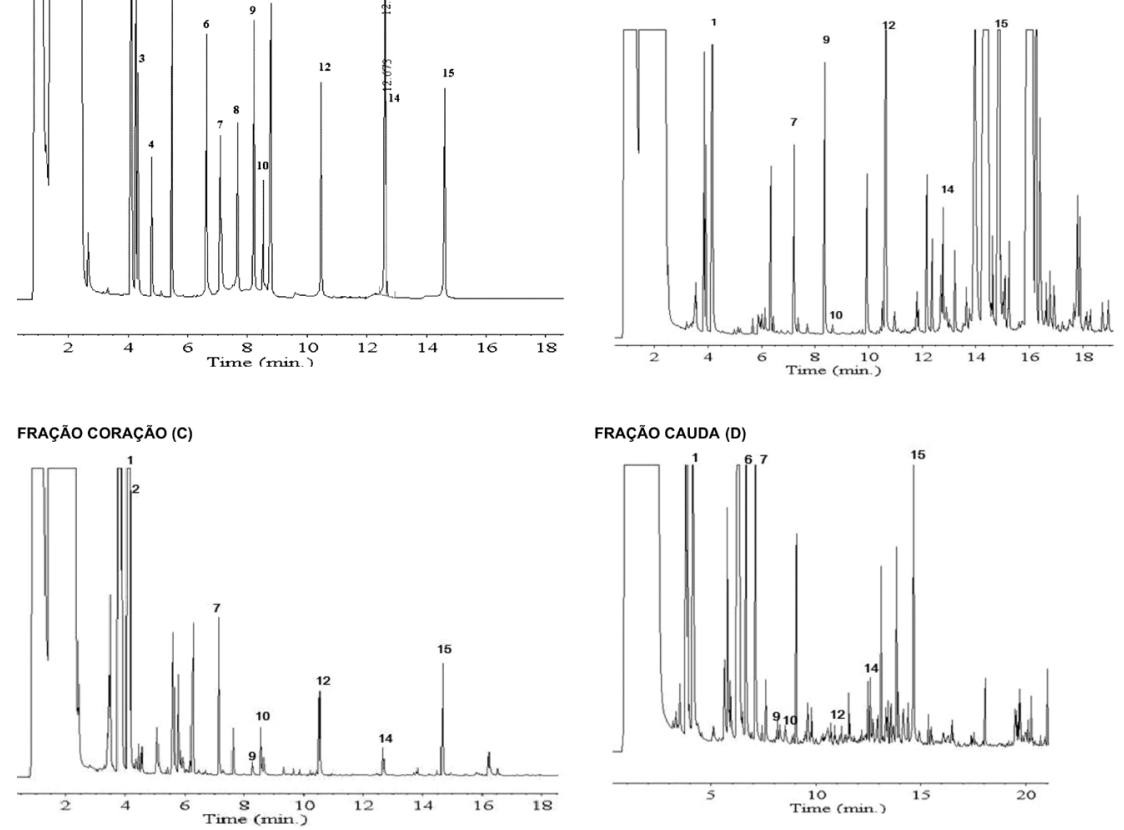

Figura 1S. Perfil cromatográfico dos 15 ácidos orgânicos derivatizados com MSTFA obtido através da análise em um CG-FID, utilizando-se uma coluna HP5, para. A programação da rampa de temperatura foi: $60^{\circ} \mathrm{C}(2 \mathrm{~min})$ até $100{ }^{\circ} \mathrm{C}$ na razão de $25^{\circ} \mathrm{C} \mathrm{min}^{-1}$ e, em seguida, para $300{ }^{\circ} \mathrm{C}$ à razão de $10{ }^{\circ} \mathrm{C} \mathrm{min}{ }^{-1}$ (5 min). $A=$ solução padrão; $B=$ fração "cabeça"; $C=$ fração "coração"; $D=$ fração "cauda" do destilado de alambique. $1=$ Ácido Lático $(\text { TMS })_{2} ; 2=$ Ácido Glicólico $(T M S)_{2} ; 3$ = Ácido Pirúvico $(T M S) ; 4=$ Ácido Oxálico $(T M S) ; 5$ = Ácido Malônico $(T M S)_{2} ; 6=$ Ácido Succínico $(\text { TMS })_{2} ; 7=$ Ácido Nonanoico (TMS) - Padrão interno; $8=$ Ácido Glutárico $(T M S)_{2} ; 9=$ Ácido Cáprico $(T M S) ; 10=$ Ácido Citramálico $(T M S)_{3} ; 11=$ Ácido Málico $(\text { TMS })_{3} ; 12=$ Ácido Láurico (TMS); 13 = Ácido Cítrico $(T M S)_{4} ; 14$ = Ácido Mirístico $(T M S)$ e 15 = Ácido Palmítico (TMS)

Tabela 1S. Concentração dos ácidos orgânicos nas amostras de aguardentes da fração coração de alambiques e de colunas oriundas do mesmo vinho

\begin{tabular}{|c|c|c|c|c|c|c|c|c|c|c|}
\hline Amostras & Classes & Ac. Lático ${ }^{a}$ & Ac. Glicólico ${ }^{\mathrm{a}}$ & Ac. Succínico & Ac. Cáprico ${ }^{\mathrm{a}}$ & Ac. Láurico & Ac. Mirístico & Ac. Palmítico & Ac. Citramálico ${ }^{a}$ & Ac. Acético \\
\hline $\mathrm{A} 30$ & coração & 3,24 & $4,00 \times 10^{-2}$ & $1,00 \times 10^{-2}$ & $<\mathrm{LD}$ & $<\mathrm{LD}$ & 0,07 & $2,30 \times 10^{-1}$ & $<\mathrm{LD}$ & 5,98 \\
\hline A31 & coração & 38,5 & $<\mathrm{LD}$ & $<\mathrm{LD}$ & $1,20 \times 10^{-1}$ & $6,00 \times 10^{-2}$ & $9,00 \times 10^{-2}$ & $3,00 \times 10^{-1}$ & $2,00 \times 10^{-2}$ & 265 \\
\hline A32 & coração & 9,21 & $2,00 \times 10^{-1}$ & $4,00 \times 10^{-2}$ & $2,60 \times 10^{-1}$ & $5,00 \times 10^{-2}$ & $8,00 \times 10^{-2}$ & $2,00 \times 10^{-1}$ & $4,00 \times 10^{-2}$ & 24,0 \\
\hline A33 & coração & 18,9 & $<\mathrm{LD}$ & $1,00 \times 10^{-2}$ & $<\mathrm{LD}$ & $5,00 \times 10^{-2}$ & $<\mathrm{LD}$ & $5,40 \times 10^{-1}$ & $<\mathrm{LD}$ & 64,8 \\
\hline A34 & coração & 35,5 & $6,90 \times 10^{-1}$ & $2,00 \times 10^{-2}$ & $<\mathrm{LD}$ & $6,00 \times 10^{-2}$ & $4,00 \times 10^{-2}$ & $3,90 \times 10^{-1}$ & $6,00 \times 10^{-2}$ & 36,7 \\
\hline $\mathrm{A} 30 \mathrm{C}$ & coluna & 2,28 & $5,00 \times 10^{-2}$ & $9,00 \times 10^{-2}$ & $<\mathrm{LD}$ & $1,00 \times 10^{-1}$ & $1,00 \times 10^{-2}$ & $1,10 \times 10^{-1}$ & $1,00 \times 10^{-2}$ & 5,34 \\
\hline $\mathrm{A} 31 \mathrm{C}$ & coluna & 54,4 & $2,00 \times 10^{-2}$ & $3,00 \times 10^{-2}$ & $<\mathrm{LD}$ & $5,00 \times 10^{-2}$ & $6,00 \times 10^{-2}$ & $6,10 \times 10^{-1}$ & $4,00 \times 10^{-2}$ & 281 \\
\hline $\mathrm{A} 32 \mathrm{C}$ & coluna & 6,36 & $<\mathrm{LD}$ & $2,00 \times 10^{-2}$ & $2,30 \times 10^{-1}$ & $5,00 \times 10^{-2}$ & $2,00 \times 10^{-2}$ & $1,20 \times 10^{-1}$ & $<\mathrm{LD}$ & 17,3 \\
\hline $\mathrm{A} 33 \mathrm{C}$ & coluna & 2,77 & $6,00 \times 10^{-2}$ & $2,00 \times 10^{-2}$ & $8,90 \times 10^{-1}$ & $1,70 \times 10^{-1}$ & $1,00 \times 10^{-2}$ & $1,10 \times 10^{-1}$ & $5,00 \times 10^{-2}$ & 5,10 \\
\hline $\mathrm{A} 34 \mathrm{C}$ & coluna & 6,05 & $4,00 \times 10^{-2}$ & $2,00 \times 10^{-2}$ & 1,22 & $2,80 \times 10^{-1}$ & $3,00 \times 10^{-2}$ & $1,40 \times 10^{-1}$ & $2,00 \times 10^{-2}$ & 5,75 \\
\hline
\end{tabular}

${ }^{\mathrm{a}}=$ As concentrações dos ácidos orgânicos foram calculadas e corrigidas, considerando-se os respectivos fatores de recuperação. 
Tabela 2S. Concentração de ácidos orgânicos (mg/100 mL de AA) nas três frações do destilado de alambique

\begin{tabular}{|c|c|c|c|c|c|c|c|c|c|c|c|}
\hline Amostras & Classes & Lático $^{\mathrm{a}}$ & Glicólico $^{\mathrm{a}}$ & Pirúvico $^{\text {a }}$ & Succinico & Cáprico $^{\mathrm{a}}$ & Láurico & Miristico & Palmitico & Citramálico $^{\mathrm{a}}$ & Acético \\
\hline A11 & cauda & 9,70 & $<\mathrm{LD}$ & $<\mathrm{LD}$ & $5,50 \times 10^{-1}$ & $<\mathrm{LD}$ & $<\mathrm{LD}$ & $<\mathrm{LD}$ & $2,60 \times 10^{-1}$ & $<\mathrm{LD}$ & 34,2 \\
\hline A12 & coração & 1,27 & $<\mathrm{LD}$ & $<\mathrm{LD}$ & $<\mathrm{LD}$ & $1,20 \times 10^{-1}$ & $6,00 \times 10^{-2}$ & $9,00 \times 10^{-2}$ & $3,00 \times 10^{-1}$ & $2,00 \times 10^{-2}$ & 30,9 \\
\hline A13 & cabeça & 3,97 & $<\mathrm{LD}$ & $<\mathrm{LD}$ & $<\mathrm{LD}$ & $9,90 \times 10^{-1}$ & $1,70 \times 10^{-1}$ & $7,00 \times 10^{-2}$ & $1,70 \times 10^{-1}$ & $<\mathrm{LD}$ & 20,2 \\
\hline A81 & cauda & 11,1 & $1,00 \times 10^{-1}$ & $2,40 \times 10^{-1}$ & $2,00 \times 10^{-2}$ & $1,70 \times 10^{-1}$ & $4,00 \times 10^{-2}$ & $8,00 \times 10^{-2}$ & $1,20 \times 10^{-1}$ & $1,40 \times 10^{-1}$ & 50,6 \\
\hline A82 & coração & 8,20 & $9,00 \times 10^{-2}$ & $<\mathrm{LD}$ & $2,00 \times 10^{-2}$ & $2,20 \times 10^{-1}$ & $6,00 \times 10^{-2}$ & $7,00 \times 10^{-2}$ & $1,80 \times 10^{-1}$ & $1,50 \times 10^{-1}$ & 64,8 \\
\hline A 83 & cabeça & 6,45 & $<\mathrm{LD}$ & $<\mathrm{LD}$ & $2,00 \times 10^{-2}$ & $1,40 \times 10^{-1}$ & $5,00 \times 10^{-2}$ & $6,00 \times 10^{-2}$ & $2,00 \times 10^{-1}$ & $8,00 \times 10^{-2}$ & 51,1 \\
\hline A201 & cauda & 46,5 & $2,70 \times 10^{-1}$ & $6,80 \times 10^{-1}$ & $8,00 \times 10^{-1}$ & $1,70 \times 10^{-1}$ & $3,00 \times 10^{-2}$ & $5,00 \times 10^{-2}$ & $2,00 \times 10^{-1}$ & $5,00 \times 10^{-2}$ & 127 \\
\hline A202 & coração & 8,85 & $<\mathrm{LD}$ & $<\mathrm{LD}$ & $1,50 \times 10^{-1}$ & $<\mathrm{LD}$ & $<\mathrm{LD}$ & $<\mathrm{LD}$ & $1,60 \times 10^{-1}$ & $<\mathrm{LD}$ & 42,4 \\
\hline A203 & cabeça & 1,41 & $<\mathrm{LD}$ & $<\mathrm{LD}$ & $5,00 \times 10^{-2}$ & 1,96 & $1,80 \times 10^{-1}$ & $3,00 \times 10^{-2}$ & $1,90 \times 10^{-1}$ & $<\mathrm{LD}$ & 15,9 \\
\hline A221 & cauda & 14,5 & $2,00 \times 10^{-2}$ & $<\mathrm{LD}$ & $6,40 \times 10^{-2}$ & $<\mathrm{LD}$ & $<\mathrm{LD}$ & $6,00 \times 10^{-2}$ & $3,40 \times 10^{-1}$ & $<\mathrm{LD}$ & 6,73 \\
\hline A222 & coração & 8,66 & $<\mathrm{LD}$ & $<\mathrm{LD}$ & $1,10 \times 10^{-1}$ & $<\mathrm{LD}$ & $<\mathrm{LD}$ & $<\mathrm{LD}$ & $1,50 \times 10^{-1}$ & $<\mathrm{LD}$ & 8,26 \\
\hline A 223 & cabeça & 2,75 & $2,30 \times 10^{-1}$ & $<\mathrm{LD}$ & $3,00 \times 10^{-2}$ & $1,40 \times 10^{-1}$ & $3,00 \times 10^{-2}$ & $2,00 \times 10^{-2}$ & $6,00 \times 10^{-2}$ & $<\mathrm{LD}$ & 3,27 \\
\hline A251 & cauda & 78,4 & $<\mathrm{LD}$ & $<\mathrm{LD}$ & $4,40 \times 10^{-1}$ & $<\mathrm{LD}$ & $<\mathrm{LD}$ & $<\mathrm{LD}$ & $4,00 \times 10^{-2}$ & $1,00 \times 10^{-1}$ & 147 \\
\hline A252 & coração & 21,9 & $<\mathrm{LD}$ & $2,00 \times 10^{-1}$ & $5,00 \times 10^{-2}$ & $<\mathrm{LD}$ & $4,00 \times 10^{-2}$ & $6,00 \times 10^{-2}$ & $3,10 \times 10^{-1}$ & $<\mathrm{LD}$ & 55,6 \\
\hline A253 & cabeça & 13,5 & $<\mathrm{LD}$ & $<\mathrm{LD}$ & $2,00 \times 10^{-2}$ & $2,00 \times 10^{-1}$ & $6,00 \times 10^{-2}$ & $5,00 \times 10^{-2}$ & $2,80 \times 10^{-1}$ & $<\mathrm{LD}$ & 27,9 \\
\hline A26 & cauda & 203 & $<\mathrm{LD}$ & $<\mathrm{LD}$ & $4,00 \times 10^{-2}$ & $2,10 \times 10^{-1}$ & $3,30 \times 10^{-1}$ & $<\mathrm{LD}$ & $1,70 \times 10^{-1}$ & $<\mathrm{LD}$ & 156 \\
\hline A262 & coração & 18,9 & $<\mathrm{LD}$ & $<\mathrm{LD}$ & $1,00 \times 10^{-2}$ & $<\mathrm{LD}$ & $5,00 \times 10^{-2}$ & $<\mathrm{LD}$ & $5,40 \times 10^{-1}$ & $<\mathrm{LD}$ & 48,0 \\
\hline A263 & cabeça & 8,63 & $<\mathrm{LD}$ & $<\mathrm{LD}$ & $<\mathrm{LD}$ & 2,35 & $2,80 \times 10^{-1}$ & $1,10 \times 10^{-1}$ & 1,05 & $3,00 \times 10^{-2}$ & 25,1 \\
\hline A301 & cauda & 32,5 & $<\mathrm{LD}$ & 1,30 & $3,00 \times 10^{-2}$ & $<\mathrm{LD}$ & $<\mathrm{LD}$ & $<\mathrm{LD}$ & $2,70 \times 10^{-1}$ & $<\mathrm{LD}$ & 43,5 \\
\hline A302 & coração & 3,24 & $4,00 \times 10^{-2}$ & $4,00 \times 10^{-1}$ & $2,00 \times 10^{-2}$ & $<\mathrm{LD}$ & $<\mathrm{LD}$ & $7,00 \times 10^{-2}$ & $2,30 \times 10^{-1}$ & $<\mathrm{LD}$ & 5,98 \\
\hline A303 & cabeça & 2,16 & $7,00 \times 10^{-2}$ & $<\mathrm{LD}$ & $2,00 \times 10^{-2}$ & $7,40 \times 10^{-1}$ & $6,00 \times 10^{-2}$ & $1,20 \times 10^{-1}$ & $4,10 \times 10^{-1}$ & $<\mathrm{LD}$ & 4,43 \\
\hline A311 & cauda & 54,6 & 8,00 & 4,98 & $5,00 \times 10^{-2}$ & $1,00 \times 10^{-1}$ & $6,00 \times 10^{-2}$ & $6,00 \times 10^{-2}$ & $2,00 \times 10^{-2}$ & $2,00 \times 10^{-2}$ & 782 \\
\hline A 312 & coração & 38,5 & $7,00 \times 10^{-2}$ & 5,70 & $<\mathrm{LD}$ & $4,00 \times 10^{-2}$ & $7,00 \times 10^{-2}$ & $7,00 \times 10^{-2}$ & $4,40 \times 10^{-1}$ & $1,40 \times 10^{-1}$ & 665 \\
\hline A 313 & cabeça & $3,05 \times 10^{-1}$ & $<\mathrm{LD}$ & $<\mathrm{LD}$ & $<\mathrm{LD}$ & $5,00 \times 10^{-1}$ & $3,40 \times 10^{-1}$ & $1,60 \times 10^{-1}$ & $7,70 \times 10^{-1}$ & $<\mathrm{LD}$ & 265 \\
\hline A 321 & cauda & 11,9 & $<\mathrm{LD}$ & $<\mathrm{LD}$ & $<\mathrm{LD}$ & $3,50 \times 10^{-1}$ & $9,00 \times 10^{-2}$ & $1,40 \times 10^{-1}$ & $1,60 \times 10^{-1}$ & $6,00 \times 10^{-2}$ & 45,1 \\
\hline A 322 & coração & 9,21 & $2,00 \times 10^{-1}$ & $<\mathrm{LD}$ & $4,00 \times 10^{-2}$ & $2,60 \times 10^{-1}$ & $5,00 \times 10^{-2}$ & $1,80 \times 10^{-2}$ & $2,00 \times 10^{-1}$ & $4,00 \times 10^{-2}$ & 24,0 \\
\hline A 323 & cabeça & 2,33 & $1,00 \times 10^{-1}$ & $<\mathrm{LD}$ & $<\mathrm{LD}$ & 1,15 & $1,30 \times 10^{-1}$ & $1,90 \times 10^{-1}$ & $6,40 \times 10^{-1}$ & $<\mathrm{LD}$ & 17,1 \\
\hline A331 & cauda & 64,2 & 1,09 & $<\mathrm{LD}$ & $5,00 \times 10^{-2}$ & $6,70 \times 10^{-1}$ & $1,00 \times 10^{-1}$ & $1,80 \times 10^{-1}$ & $3,60 \times 10^{-1}$ & $3,30 \times 10^{-1}$ & 53,0 \\
\hline A332 & coração & 13,0 & $2,30 \times 10^{-1}$ & $<\mathrm{LD}$ & $2,00 \times 10^{-2}$ & $4,60 \times 10^{-1}$ & $1,30 \times 10^{-1}$ & $7,00 \times 10^{-2}$ & $3,40 \times 10^{-1}$ & $8,00 \times 10^{-2}$ & 15,5 \\
\hline A333 & cabeça & 2,98 & $1,70 \times 10^{-1}$ & $2,00 \times 10^{-2}$ & $2,00 \times 10^{-2}$ & 3,49 & $2,80 \times 10^{-1}$ & $1,20 \times 10^{-1}$ & $3,80 \times 10^{-1}$ & $2,00 \times 10^{-2}$ & 9,22 \\
\hline A 341 & cauda & 97,5 & 1,51 & $<\mathrm{LD}$ & $5,00 \times 10^{-2}$ & $<\mathrm{LD}$ & $1,10 \times 10^{-1}$ & $1,80 \times 10^{-1}$ & $1,30 \times 10^{-1}$ & $1,80 \times 10^{-1}$ & 74,7 \\
\hline A342 & coração & 35,5 & $7,00 \times 10^{-1}$ & $<\mathrm{LD}$ & $2,00 \times 10^{-2}$ & $<\mathrm{LD}$ & $6,00 \times 10^{-2}$ & $4,00 \times 10^{-2}$ & $4,00 \times 10^{-1}$ & $6,00 \times 10^{-2}$ & 36,7 \\
\hline A 343 & cabeça & 12,0 & $<\mathrm{LD}$ & $<\mathrm{LD}$ & $3,00 \times 10^{-2}$ & 10,4 & 1,62 & $4,10 \times 10^{-1}$ & 1,60 & $5,00 \times 10^{-2}$ & 8,02 \\
\hline A371 & cauda & 62,25 & $<\mathrm{LD}$ & $<\mathrm{LD}$ & $<\mathrm{LD}$ & $<\mathrm{LD}$ & $3,00 \times 10^{-2}$ & $8,00 \times 10^{-1}$ & $3,40 \times 10^{-1}$ & $6,00 \times 10^{-2}$ & 69,7 \\
\hline A 372 & coração & 16,19 & $7,00 \times 10^{-2}$ & $<\mathrm{LD}$ & $<\mathrm{LD}$ & $<\mathrm{LD}$ & $1,20 \times 10^{-1}$ & $8,00 \times 10^{-1}$ & $8,10 \times 10^{-1}$ & $2,00 \times 10^{-2}$ & 43,5 \\
\hline A 373 & cabeça & 7,10 & $1,80 \times 10^{-1}$ & $<\mathrm{LD}$ & $1,00 \times 10^{-2}$ & 2,37 & $7,30 \times 10^{-1}$ & $2,80 \times 10^{-2}$ & 2,02 & $<\mathrm{LD}$ & 58,31 \\
\hline D051 & cauda & 3,61 & $1,20 \times 10^{-1}$ & $<\mathrm{LD}$ & $4,00 \times 10^{-2}$ & 3,03 & $3,30 \times 10^{-1}$ & $<\mathrm{LD}$ & $4,00 \times 10^{-2}$ & $1,70 \times 10^{-1}$ & $<\mathrm{LD}$ \\
\hline D052 & coração & $7,20 \times 10^{-1}$ & $<\mathrm{LD}$ & 3,61 & $2,00 \times 10^{-2}$ & 1,19 & $1,80 \times 10^{-1}$ & $5,00 \times 10^{-2}$ & $3,30 \times 10^{-1}$ & $4,00, \times 10^{-2}$ & 21,88 \\
\hline D053 & cabeça & $2,81 \times 10^{-1}$ & $<\mathrm{LD}$ & $<\mathrm{LD}$ & $2,00 \times 10^{-2}$ & $4,10 \times 10^{-1}$ & $9,10 \times 10^{-1}$ & $1,80 \times 10^{-1}$ & $6,40 \times 10^{-1}$ & $3,00 \times 10^{-2}$ & 616 \\
\hline D061 & cauda & 3,03 & $<\mathrm{LD}$ & 0,27 & $3,00 \times 10^{-2}$ & 2,51 & $2,78 \times 10^{-1}$ & $<\mathrm{LD}$ & $2,00 \times 10^{-2}$ & $9,00 \times 10^{-2}$ & $<\mathrm{LD}$ \\
\hline D062 & coração & $6,83 \times 10^{-1}$ & $2,00 \times 10^{-2}$ & $<\mathrm{LD}$ & $2,00 \times 10^{-2}$ & 2,89 & $2,78 \times 10^{-1}$ & $7,00 \times 10^{-2}$ & $2,50 \times 10^{-1}$ & $1,00 \times 10^{-1}$ & 28,49 \\
\hline D063 & cabeça & $4,11 \times 10^{-1}$ & $<\mathrm{LD}$ & $<\mathrm{LD}$ & $2,00 \times 10^{-2}$ & 10,1 & 1,05 & $2,30 \times 10^{-1}$ & 1,02 & $3,00 \times 10^{-2}$ & 52,66 \\
\hline ACLO1 & cauda & 15,2 & $<\mathrm{LD}$ & $<\mathrm{LD}$ & $<\mathrm{LD}$ & $<\mathrm{LD}$ & $1,20 \times 10^{-2}$ & $8,00 \times 10^{-2}$ & $7,00 \times 10^{-2}$ & $5,00 \times 10^{-2}$ & $<\mathrm{LD}$ \\
\hline ACLO2 & coração & 6,12 & $<\mathrm{LD}$ & $<\mathrm{LD}$ & $<\mathrm{LD}$ & $4,30 \times 10^{-1}$ & $1,20 \times 10^{-1}$ & $1,00 \times 10^{-1}$ & $6,70 \times 10^{-1}$ & $2,00 \times 10^{-2}$ & 300 \\
\hline ACLO3 & cabeça & 2,68 & $<\mathrm{LD}$ & $<\mathrm{LD}$ & $<\mathrm{LD}$ & 5,52 & 1,51 & $4,10 \times 10^{-1}$ & 2,58 & $2,00 \times 10^{-2}$ & 53,0 \\
\hline
\end{tabular}

${ }^{a}=$ As concentrações dos ácidos foram calculadas e corrigidas, considerando-se os respectivos fatores de recuperação. 
Tabela 3S. Valores médios e de mediana dos quatro descritores das amostras correspondentes a cada fração do destilado de alambique e dos seus outliers

\begin{tabular}{|c|c|c|c|c|}
\hline \multicolumn{5}{|c|}{ Ácidos Orgânicos } \\
\hline Fração "Cabeça" & Lático & Láurico & Mirístico & Pamítico \\
\hline A203 & 1,41 & $1,80 \times 10^{-1}$ & $3,00 \times 10^{-2}$ & $1,90 \times 10^{-1}$ \\
\hline A253 & 8,63 & $6,00 \times 10^{-2}$ & $5,00 \times 10^{-2}$ & $2,80 \times 10^{-1}$ \\
\hline A263 & 4,57 & $2,80 \times 10^{-1}$ & $1,10 \times 10^{-1}$ & 1,05 \\
\hline A303 & 2,16 & $6,00 \times 10^{-2}$ & $1,20 \times 10^{-1}$ & $4,10 \times 10^{-1}$ \\
\hline A313 & 2,33 & $3,40 \times 10^{-1}$ & $1,60 \times 10^{-1}$ & $7,70 \times 10^{-1}$ \\
\hline A323 & 12,0 & $1,30 \times 10^{-1}$ & $1,90 \times 10^{-1}$ & $6,40 \times 10^{-1}$ \\
\hline A343 & 7,10 & 1,62 & $4,10 \times 10^{-1}$ & 1,60 \\
\hline A373 & 8,63 & $7,30 \times 10^{-1}$ & $2,80 \times 10^{-1}$ & 2,02 \\
\hline Média & 5,85 & $4,30 \times 10^{-1}$ & $1,70 \times 10^{-1}$ & $8,70 \times 10^{-1}$ \\
\hline Mediana & 5,84 & $2,30 \times 10^{-1}$ & $1,40 \times 10^{-1}$ & $7,10 \times 10^{-1}$ \\
\hline \multicolumn{5}{|l|}{ Fração“Coração” } \\
\hline A 12 & 1,27 & $6,00 \times 10^{-2}$ & $9,00 \times 10^{-2}$ & $3,00 \times 10^{-1}$ \\
\hline A82 & 8,18 & $6,00 \times 10^{-2}$ & $1,80 \times 10^{-1}$ & $1,80 \times 10^{-1}$ \\
\hline A202 & 8,85 & $4,00 \times 10^{-2}$ & $1,60 \times 10^{-1}$ & $1,60 \times 10^{-1}$ \\
\hline A 222 & 8,65 & $5,00 \times 10^{-2}$ & $<\mathrm{LD}$ & $3,10 \times 10^{-1}$ \\
\hline A252 & 21,9 & $1,30 \times 10^{-1}$ & $6,00 \times 10^{-2}$ & $5,40 \times 10^{-1}$ \\
\hline A262 & 18,9 & $1,20 \times 10^{-1}$ & $<\mathrm{LD}$ & $2,30 \times 10^{-1}$ \\
\hline A302 & 3,24 & $1,80 \times 10^{-1}$ & $7,00 \times 10^{-2}$ & $3,40 \times 10^{-1}$ \\
\hline A332 & 9,21 & $2,80 \times 10^{-1}$ & $7,00 \times 10^{-2}$ & $8,10 \times 10^{-1}$ \\
\hline A372 & 13,0 & $1,20 \times 10^{-1}$ & $8,00 \times 10^{-2}$ & $3,30 \times 10^{-1}$ \\
\hline D052 & 16,1 & $6,00 \times 10^{-2}$ & $5,00 \times 10^{-2}$ & $2,50 \times 10^{-1}$ \\
\hline D062 & $7,20 \times 10^{-1}$ & $6,00 \times 10^{-2}$ & $7,00 \times 10^{-2}$ & $6,70 \times 10^{-1}$ \\
\hline ACLO2 & $6,80 \times 10^{-1}$ & $4,00 \times 10^{-2}$ & $1,00 \times 10^{-1}$ & $3,00 \times 10^{-1}$ \\
\hline Média & 9,24 & $1,00 \times 10^{-1}$ & $8,00 \times 10^{-2}$ & $3,30 \times 10^{-1}$ \\
\hline Mediana & 8,75 & $6,00 \times 10^{-2}$ & $7,00 \times 10^{-2}$ & $3,00 \times 10^{-1}$ \\
\hline \multicolumn{5}{|l|}{ Fração "Cauda" } \\
\hline A201 & 46,5 & $3,00 \times 10^{-2}$ & $5,00 \times 10^{-2}$ & $2,00 \times 10^{-1}$ \\
\hline A251 & 78,4 & $<\mathrm{LD}$ & $<\mathrm{LD}$ & $4,00 \times 10^{-1}$ \\
\hline A261 & 203 & $3,30 \times 10^{-1}$ & $<\mathrm{LD}$ & $1,70 \times 10^{-1}$ \\
\hline A301 & 32,5 & $<\mathrm{LD}$ & $<\mathrm{LD}$ & $2,70 \times 10^{-1}$ \\
\hline A311 & 54,6 & $6,00 \times 10^{-2}$ & $1,80 \times 10^{-1}$ & $2,00 \times 10^{-2}$ \\
\hline A331 & 64,2 & $1,00 \times 10^{-1}$ & $1,80 \times 10^{-1}$ & $3,60 \times 10^{-1}$ \\
\hline A341 & 46,5 & $1,10 \times 10^{-1}$ & $8,00 \times 10^{-2}$ & $1,30 \times 10^{-1}$ \\
\hline A371 & 78,4 & $3,00 \times 10^{-2}$ & $5,00 \times 10^{-2}$ & $3,40 \times 10^{-1}$ \\
\hline Média & 75,0 & $8,00 \times 10^{-2}$ & $7,00 \times 10^{-2}$ & $1,90 \times 10^{-1}$ \\
\hline Mediana & 54,6 & $4,00 \times 10^{-2}$ & $5,00 \times 10^{-2}$ & $1,90 \times 10^{-1}$ \\
\hline
\end{tabular}

Tabela 3S. Continuação

\begin{tabular}{|c|c|c|c|c|}
\hline $\begin{array}{l}\text { Outliers na fração } \\
\text { coração referente a: }\end{array}$ & Lático & Láurico & Mirístico & Pamítico \\
\hline \multicolumn{5}{|l|}{ Fração "Cabeça" } \\
\hline A13 & 3,97 & $1,70 \times 10^{-1}$ & $7,00 \times 10^{-2}$ & $1,70 \times 10^{-1}$ \\
\hline A83 & 6,45 & $5,00 \times 10^{-2}$ & $6,00 \times 10^{-2}$ & $2,00 \times 10^{-1}$ \\
\hline A203 & 2,75 & $1,80 \times 10^{-1}$ & $3,00 \times 10^{-2}$ & $1,90 \times 10^{-1}$ \\
\hline A223 & 13,5 & $3,00 \times 10^{-2}$ & $2,00 \times 10^{-2}$ & $6,00 \times 10^{-2}$ \\
\hline A253 & 2,16 & $6,00 \times 10^{-2}$ & $5,00 \times 10^{-2}$ & $2,80 \times 10^{-1}$ \\
\hline A303 & 2,98 & $2,80 \times 10^{-1}$ & $1,20 \times 10^{-1}$ & $4,10 \times 10^{-1}$ \\
\hline A333 & 3,97 & $1,70 \times 10^{-1}$ & $1,20 \times 10^{-1}$ & $3,80 \times 10^{-1}$ \\
\hline Média & 5,10 & $1,30 \times 10^{-1}$ & $7,00 \times 10^{-2}$ & $2,40 \times 10^{-1}$ \\
\hline Mediana & 3,97 & $1,70 \times 10^{-1}$ & $6,00 \times 10^{-2}$ & $2,20 \times 10^{-1}$ \\
\hline \multicolumn{5}{|l|}{ Fração "Cauda" } \\
\hline A11 & 9,70 & $<\mathrm{LD}$ & $<\mathrm{LD}$ & $2,60 \times 10^{-1}$ \\
\hline A81 & 11,1 & $4,00 \times 10^{-2}$ & $8,00 \times 10^{-2}$ & $1,20 \times 10^{-1}$ \\
\hline A221 & 14,5 & $<\mathrm{LD}$ & $6,00 \times 10^{-2}$ & $3,40 \times 10^{-1}$ \\
\hline A321 & 11,8 & $9,00 \times 10^{-2}$ & $1,40 \times 10^{-1}$ & $1,60 \times 10^{-1}$ \\
\hline D051 & 3,61 & $3,30 \times 10^{-1}$ & $<\mathrm{LD}$ & $4,00 \times 10^{-2}$ \\
\hline D061 & 3,03 & $2,30 \times 10^{-1}$ & $<\mathrm{LD}$ & $2,00 \times 10^{-2}$ \\
\hline ACLO1 & 15,2 & $1,00 \mathrm{v}$ & $8,00 \times 10^{-2}$ & $7,00 \times 10^{-2}$ \\
\hline Média & 9,84 & $1,50 \times 10^{-1}$ & $5,00 \times 10^{-2}$ & $1,40 \times 10^{-1}$ \\
\hline Mediana & 11,1 & $1,00 \times 10^{-1}$ & $6,00 \times 10^{-2}$ & $1,20 \times 10^{-1}$ \\
\hline \multicolumn{5}{|l|}{ Fração “Coração" } \\
\hline A312 & 38,5 & $6,00 \times 10^{-2}$ & $7,00 \times 10^{-2}$ & $4,40 \times 10^{-1}$ \\
\hline A342 & 35,5 & $6,00 \times 10^{-2}$ & $4,00 \times 10^{-2}$ & $4,00 \times 10^{-1}$ \\
\hline Média & 37,0 & $6,00 \times 10^{-2}$ & $5,50 \times 10^{-2}$ & $4,2 \times 10^{-1}$ \\
\hline Mediana & 37,0 & $6,00 \times 10^{-2}$ & $6,50 \times 10^{-2}$ & $4,2 \times 10^{-1}$ \\
\hline
\end{tabular}

